\title{
Uncertain initial imperfections via probabilistic and convex modeling: axial impact buckling of a clamped beam
}

\author{
Wim Verhaeghe ${ }^{\mathrm{a}, *}$, Isaac Elishakoff ${ }^{\mathrm{b}}$, Wim Desmet $^{\mathrm{a}}$, Dirk Vandepitte ${ }^{\mathrm{a}}$, David \\ Moens $^{\text {a,c }}$ \\ ${ }^{a}$ KU Leuven, Dept. of Mechanical Engineering, Celestijnenlaan 300B, B-3001 Heverlee, \\ Belgium \\ ${ }^{b}$ Florida Atlantic University, Boca Raton, Florida 33431, USA \\ ${ }^{c}$ Lessius Mechelen, Campus De Nayer, Dept. of Applied Engineering, KU Leuven \\ Association, J. De Nayerlaan 5, B-2860, Sint-Katelijne-Waver, Belgium
}

\begin{abstract}
In the present study the problem of axial impact of a clamped beam with initial imperfections is treated using two uncertainty descriptions. For the deterministic solution, the exact mode and an approximative approach using so called "cosinusoidal polynomials" by Filonenko-Borodich are used. Next, the uncertainty is represented by a convex set and subsequently by random variables. The comparison between both uncertainty descriptions is carried out numerically on the level of the design time of the considered beam.
\end{abstract}

Keywords: uncertainty modeling; time to failure; exact mode shapes; cosinusoidal polynomials

*wim.verhaeghe@mech.kuleuven.be, (Tel.) +32 16 328606, (Fax.) +32 16322987 , Afdeling PMA, Kasteelpark Arenberg 41, bus 2449, B-3001 Heverlee, Belgium 


\section{Nomenclature}

$$
\begin{aligned}
& E \quad=\text { Young's modulus } \\
& I=\text { moment of inertia } \\
& P \quad=\text { axial load } \\
& \rho \quad=\text { mass density } \\
& A \quad=\text { cross-sectional area } \\
& x \quad=\text { axial coordinate } \\
& t \quad=\text { time } \\
& \bar{y}(x) \quad=\quad \text { initial imperfection } \\
& y(x) \quad=\text { additional transverse deflection measured form } \bar{y}(x) \\
& \xi \quad=\text { non-dimensional axial coordinate } \\
& \tau=\text { non-dimensional time } \\
& \alpha=\text { non-dimensional axial load } \\
& \bar{u}(\xi)=\text { non-dimensional initial displacement } \\
& u(\xi, \tau)=\text { non-dimensional additional transverse deflection measured from } \bar{u}(\xi) \\
& P_{C L} \quad=\text { classical buckling load of a perfect beam clamped at both ends } \\
& \omega_{1} \quad=\text { fundamental eigenfrequency of beam clamped at both ends } \\
& \text { in the absence of axial compression } \\
& \Delta \quad=\quad \text { radius of gyration of the beam } \\
& H_{i} \quad=\quad \text { uncertain amplitudes of initial imperfections co-configurational } \\
& \text { with exact mode shapes } \\
& P_{i}(\xi) \quad=\text { exact mode shape } i \text { of beam without axial loading } \\
& A_{i} \quad=\text { non-dimensional uncertain amplitudes corresponding to exact } \\
& \text { mode shapes } \\
& H_{i}^{*} \quad=\quad \text { uncertain amplitudes corresponding to terms of } \\
& \text { Filonenko-Borodich series } \\
& P_{i}^{*}(\xi) \quad=\quad \text { term } i \text { of Filonenko-Borodich series } \\
& A_{i}^{*} \quad=\text { non-dimensional uncertain amplitudes corresponding to terms of } \\
& \text { Filonenko-Borodich series } \\
& \nu(\xi, \tau)=\text { non-dimensional total displacement } \\
& Z(\theta, W)=\text { ellipse defined by matrix } W \text { and size-parameter } \theta \\
& \lambda=\text { Lagrangian multiplier } \\
& \delta(\tau)=\text { integral (span-averaged) displacement along the beam at time } \tau
\end{aligned}
$$




\section{Introduction}

Dynamic impact buckling of structures with initial imperfections has been a field of study for several decades. A well investigated one-mass structure was introduced by Hutchinson and Budiansky [11]. The structure consisted in a three-hinge, rigid-rod model that was laterally constrained by a nonlinear softening spring, and a mass $M$ concentrated at the hinge joining the two rods. The initial imperfection is modelled by one single parameter identified with the deviation of the unloaded structure from the straight configuration. Danielson [4] compared the results of this simple model with a spherical shell under a step loading of external pressure. He used an analytical solution method based on a singular perturbation technique. Kounadis has introduced a comprehensive dynamic buckling analysis for two-degree-of-freedom systems in [12].

Cylindrical shells under transient lateral pressures were studied by Anderson and Lindberg [1] to formulate a theory covering the full range of loadings from short impulse to quasi-static. A comparison of beam impact models for transverse loadings was carried out by Garrelick and Benveniste [8].

Blackketter and May [3] describe an analytical method to study the permanent deformation of an axial rod subjected to dynamic loading. They did not consider buckling, but represented the material by a parametrized nonlinear stress-strain relationship and applied the Bubnov-Galerkin method to find the permanent deformation.

The study of the axial impact buckling of beams with simply supported boundary conditions and uncertain initial imperfections has been delt with by Lindberg [13], Elishakoff [5, 6] and Ben-Haim and Elishakoff [2] and other authors.

Lindberg [13] apparently was the first investigator who pioneered probabilistic treatment of initial imperfections in the impact problems of a beam. He 
resolved the initial imperfections and displacements in series with respect to natural mode shapes, with initial imperfection amplitudes turning out to be the random variables. He assumed that the initial imperfections were a homogeneous random field and used alternate zero crossings approach.

Elishakoff [5] provided a closed form solution for a beam whose initial imperfection is proportional to the fundamental mode shape. He used Hoff's [9] criterion of stability, which states that failure is presumed to occur if the instantaneous displacement exceeds a specified threshold. In the following study Elishakoff [6] extended his approach to include multiple random variables in initial imperfections, and treated the problem via Monte Carlo Method.

Ben-Haim and Elishakoff [2] study the above problem in a non-probabilistic way, that is to treat the uncertain initial imperfections by a convex model. The calculation of a number of bounds is carried out: maximum deflection, duration above a threshold and maximum integral displacement. Closed-form solutions are obtained for these bounds. Latest works in this direction are by $[16,19,10]$. Lindberg comments on the comparison of the convex and probabilistic model in [14]. It is shown that a clear relationship can be derived between the standard deviation and the maximum displacement in the probabilistic and the convex analysis, respectively.

The effect of boundary conditions was not investigated in the above uncertain initial imperfection studies. In the present study the problem of impact buckling of a clamped beam (see Figure 1) with uncertain initial imperfections is treated both by convex modelling and probabilistic analysis. Two solution methods are presented: one based on the exact mode shapes of a clamped beam and one utilizing so called "cosinusoidal polynomials" by Filonenko-Borodich [7] as the set of comparison functions within the Bubnov-Galerkin methodology. 


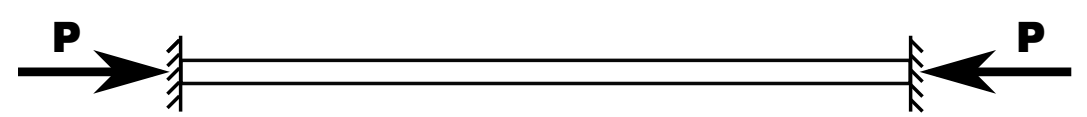

Figure 1: The bi-clamped beam with axial impact loading.

\section{Governing equation}

Following [15], we disregard axial wave propagation and assume uniform compression throughout the beam, whose motion obeys the differential equation:

$$
E I \frac{\partial^{4} y}{\partial x^{4}}+P \frac{\partial^{2} y}{\partial x^{2}}+\rho A \frac{\partial^{2} y}{\partial t^{2}}=-P \frac{\partial^{2} \bar{y}}{\partial x^{2}}
$$

$E I$ and $\rho A$ are taken as constant.

For the clamped-clamped beam, the differential equation (1) is supplemented by the boundary conditions

$$
y(x, t)=0, \quad \frac{\partial y(x, t)}{\partial x}=0 ; \quad \text { at } x=0, l
$$

and the initial conditions

$$
y(x, t)=0, \quad \frac{\partial y(x, t)}{\partial t}=0 ; \quad \text { at } t=0
$$

The excitation constitutes a compressive step-loading: The constant load $P$ is applied at time $\tau=0$ and kept indefinitely.

\section{Solution of the deterministic problem}

For the deterministic solution of the problem we introduce following nondimensional quantities

$$
\xi=\frac{x}{l}, \quad \tau=\omega_{1} t, \quad \alpha=\frac{P}{P_{C L}}, \quad u(\xi, \tau)=\frac{y(x, t)}{\Delta}, \quad \bar{u}(\xi)=\frac{\bar{y}(x)}{\Delta}
$$


where

$$
P_{C L}=\frac{4 \pi^{2} E I}{l^{2}}, \quad \omega_{1}=\gamma\left(\frac{\pi}{l}\right)^{2} \sqrt{\frac{E I}{\rho A}}, \quad \Delta=\sqrt{\frac{I}{A}}
$$

$\gamma=4.73^{2} / \pi^{2}=2.27$ according to Ref. [17].

Equation (1) becomes

$$
\frac{\partial^{4} u}{\partial \xi^{4}}+4 \pi^{2} \alpha \frac{\partial^{2} u}{\partial \xi^{2}}+\gamma^{2} \pi^{4} \frac{\partial^{2} u}{\partial \tau^{2}}=-4 \pi^{2} \alpha \frac{\partial^{2} \bar{u}}{\partial \xi^{2}}
$$

3.1. Initial imperfection that is co-configurational with the first two exact mode shapes

For the solution method using the exact mode shape, the shape of the imperfection is taken as follows

$$
\bar{y}(x)=H_{0} P_{0}\left(\frac{x}{l}\right)+H_{1} P_{1}\left(\frac{x}{l}\right)
$$

where

$$
\begin{aligned}
P_{0}\left(\frac{x}{l}\right)= & \cos \left(\sqrt{\gamma} \pi \frac{x}{l}\right)-\cosh \left(\sqrt{\gamma} \pi \frac{x}{l}\right)-\ldots \\
& \frac{\cos (\sqrt{\gamma} \pi)-\cosh (\sqrt{\gamma} \pi)}{\sin (\sqrt{\gamma} \pi)-\sinh (\sqrt{\gamma} \pi)}\left[\sin \left(\sqrt{\gamma} \pi \frac{x}{l}\right)-\sinh \left(\sqrt{\gamma} \pi \frac{x}{l}\right)\right] \\
P_{1}\left(\frac{x}{l}\right)= & \cos \left(\sqrt{\zeta} \pi \frac{x}{l}\right)-\cosh \left(\sqrt{\zeta} \pi \frac{x}{l}\right)-\ldots \\
& \frac{\cos (\sqrt{\zeta} \pi)-\cosh (\sqrt{\zeta} \pi)}{\sin (\sqrt{\zeta} \pi)-\sinh (\sqrt{\zeta} \pi)}\left[\sin \left(\sqrt{\zeta} \pi \frac{x}{l}\right)-\sinh \left(\sqrt{\zeta} \pi \frac{x}{l}\right)\right]
\end{aligned}
$$

with $\gamma=4.73^{2} / \pi^{2}=2.27$ and $\zeta=7.85^{2} / \pi^{2}=6.25$. These constitute the first two exact mode shapes of the beam without axial loading [17]. After nondimensionalizing, Eq. (7) becomes

$$
\bar{u}(\xi)=A_{0} P_{0}(\xi)+A_{1} P_{1}(\xi)
$$


with $A_{i}=H_{i} / \Delta, i=0,1$. The boundary conditions which follow from substituting (10) in (6) are satisfied by setting:

$$
u(\xi, \tau)=G_{0}(\tau) P_{0}(\xi)+G_{1}(\tau) P_{1}(\xi)
$$

For $P_{0}(\xi)$ and $P_{1}(\xi)$ the following relations holds:

$$
\frac{d^{4} P_{0}(\xi)}{d \xi^{4}}=\gamma^{2} \pi^{4} P_{0}(\xi), \quad \frac{d^{4} P_{1}(\xi)}{d \xi^{4}}=\zeta^{2} \pi^{4} P_{1}(\xi)
$$

Combining Eqs. (6),(11) and (12) leads to

$$
\begin{aligned}
& \gamma^{2} \pi^{4} P_{0}(\xi) G_{0}(\tau)+\zeta^{2} \pi^{4} P_{1}(\xi) G_{1}(\tau)+4 \pi^{2} \alpha \frac{\partial^{2} P_{0}(\xi)}{\partial \xi^{2}} G_{0}(\tau)+\ldots \\
& 4 \pi^{2} \alpha \frac{\partial^{2} P_{1}(\xi)}{\partial \xi^{2}} G_{1}(\tau)+\gamma^{2} \pi^{4} P_{0}(\xi) \frac{\partial^{2} G_{0}(\tau)}{\partial \tau^{2}}+\gamma^{2} \pi^{4} P_{1}(\xi) \frac{\partial^{2} G_{1}(\tau)}{\partial \tau^{2}}=\ldots \\
& \quad-4 \pi^{2} \alpha A_{0} \frac{\partial^{2} P_{0}(\xi)}{\partial \xi^{2}}-4 \pi^{2} \alpha A_{1} \frac{\partial^{2} P_{1}(\xi)}{\partial \xi^{2}}
\end{aligned}
$$

Because of following relations,

$$
\begin{aligned}
& \int_{0}^{1} P_{0}(\xi) P_{0}(\xi) d \xi=1, \quad \int_{0}^{1} P_{1}(\xi) P_{1}(\xi) d \xi=1 \\
& \int_{0}^{1} P_{0}(\xi) \frac{\partial^{2} P_{0}(\xi)}{\partial \xi^{2}}=-\kappa \frac{\pi^{2}}{4}, \quad \kappa=4.99 \\
& \int_{0}^{1} P_{1}(\xi) \frac{\partial^{2} P_{1}(\xi)}{\partial \xi^{2}}=-\eta \frac{\pi^{2}}{4}, \quad \eta=18.66 \\
& \int_{0}^{1} P_{0}(\xi) P_{1}(\xi) d \xi=0, \quad \int_{0}^{1} P_{0}(\xi) \frac{\partial^{2} P_{1}(\xi)}{\partial \xi^{2}}=0, \quad \int_{0}^{1} P_{1}(\xi) \frac{\partial^{2} P_{0}(\xi)}{\partial \xi^{2}}=0
\end{aligned}
$$

the multiplication of (13) with $P_{0}(\xi)$ and subsequent integration over $\xi=0 \ldots 1$ leads to following ordinary differential equation for $G_{0}(\tau)$

$$
\frac{d^{2} G_{0}(\tau)}{d \tau^{2}}+G_{0}(\tau)\left(1-\frac{\kappa}{\gamma^{2}} \alpha\right)=\frac{\kappa}{\gamma^{2}} \alpha A_{0}
$$


and multiplication of (13) with $P_{1}(\xi)$ and subsequent integration over $\xi=0 \ldots 1$ leads to following ordinary differential equation for $G_{1}(\tau)$

$$
\frac{d^{2} G_{1}(\tau)}{d \tau^{2}}+G_{1}(\tau)\left(\frac{\zeta^{2}}{\gamma^{2}}-\frac{\eta}{\gamma^{2}} \alpha\right)=\frac{\eta}{\gamma^{2}} \alpha A_{1}
$$

The solution of Eq. (16) reads $G_{0}(\tau)=A_{0} \psi_{0}(\tau)$ with

$$
\psi_{0}(\tau)= \begin{cases}\frac{\kappa \alpha}{\kappa \alpha-\gamma^{2}}\left[\cos \left(\tau \sqrt{1-\frac{\kappa}{\gamma^{2}} \alpha}\right)-1\right] & \text { if } \alpha<\frac{\gamma^{2}}{\kappa} \\ \frac{\tau^{2}}{2} & \text { if } \alpha=\frac{\gamma^{2}}{\kappa} \\ \frac{\kappa \alpha}{\kappa \alpha-\gamma^{2}}\left[\cosh \left(\tau \sqrt{\frac{\kappa}{\gamma^{2}} \alpha-1}\right)-1\right] & \text { if } \alpha>\frac{\gamma^{2}}{\kappa}\end{cases}
$$

whereas the solution of equation (17) reads $G_{1}(\tau)=A_{1} \psi_{1}(\tau)$, where

$$
\psi_{1}(\tau)= \begin{cases}\frac{\eta \alpha}{\eta \alpha-\zeta^{2}}\left[\cos \left(\tau \sqrt{\frac{\zeta^{2}}{\gamma^{2}}-\frac{\eta}{\gamma^{2}} \alpha}\right)-1\right] & \text { if } \alpha<\frac{\zeta^{2}}{\eta} \\ \frac{\zeta^{2}}{\gamma^{2}} \frac{\tau^{2}}{2} & \text { if } \alpha=\frac{\zeta^{2}}{\eta} \\ \frac{\eta \alpha}{\eta \alpha-\zeta^{2}}\left[\cosh \left(\tau \sqrt{\frac{\eta}{\gamma^{2}} \alpha-\frac{\zeta^{2}}{\gamma^{2}}}\right)-1\right] & \text { if } \alpha>\frac{\zeta^{2}}{\eta}\end{cases}
$$

The total displacement is thus described by:

$$
\nu(\xi, \tau)=u(\xi, \tau)+\bar{u}(\xi)=A_{0}\left[1+\psi_{0}(\tau)\right] P_{0}(\xi)+A_{1}\left[1+\psi_{1}(\tau)\right] P_{1}(\xi)
$$

Usually the problems are solved via approximate techniques due to possible inhomogeneity or other deviations from uniformity. In order to model the effect of approximate techniques, we provide hereinafter the solution via the Bubnov-Galerkin method utilizing, as a comparison function, so called 
Filonenko-Borodich "cosinusoidal polynomials".

\subsection{Solution using two terms of Filonenko-Borodich series}

In the approximate solution, the shape of the imperfection is taken as follows

$$
\bar{y}=H_{0}^{*} P_{0}^{*}\left(\frac{x}{l}\right)+H_{1}^{*} P_{1}^{*}\left(\frac{x}{l}\right)
$$

where

$$
P_{0}^{*}\left(\frac{x}{l}\right)=1-\cos \left(2 \pi \frac{x}{l}\right), \quad P_{1}^{*}\left(\frac{x}{l}\right)=\cos \left(\pi \frac{x}{l}\right)-\cos \left(3 \pi \frac{x}{l}\right)
$$

are the first two terms in the so called "cosinusoidal polynomials" by FilonenkoBorodich [7]. Some mathematical properties of these functions were proved by Vilenkin [18]. After nondimensionalizing, Eq. (21) becomes

$$
\bar{u}(\xi)=A_{0}^{*}[1-\cos (2 \pi \xi)]+A_{1}^{*}[\cos (\pi \xi)-\cos (3 \pi \xi)], \quad A_{i}^{*}=\frac{H_{i}^{*}}{\Delta} \quad i=0,1
$$

The boundary conditions which follow from substituting (23) in (6) are satisfied by setting:

$$
u(\xi, \tau)=G_{0}^{*}(\tau) P_{0}^{*}(\xi)+G_{1}^{*}(\tau) P_{1}^{*}(\xi)
$$

The error of this solution equals

$$
\begin{aligned}
\epsilon(\xi, \tau)= & G_{0}^{*}(\tau) \frac{d^{4} P_{0}^{*}(\xi)}{d \xi^{4}}+G_{1}^{*}(\tau) \frac{d^{4} P_{1}^{*}(\xi)}{d \xi^{4}}+4 \pi^{2} \alpha G_{0}^{*}(\tau) \frac{d^{2} P_{0}^{*}(\xi)}{d \xi^{2}}+\ldots \\
& 4 \pi^{2} \alpha G_{1}^{*}(\tau) \frac{d^{2} P_{1}^{*}(\xi)}{d \xi^{2}}+\gamma^{2} \pi^{4} P_{0}^{*}(\xi) \frac{\partial^{2} G_{0}^{*}(\tau)}{\partial \tau^{2}}+\gamma^{2} \pi^{4} P_{1}^{*}(\xi) \frac{\partial^{2} G_{1}^{*}(\tau)}{\partial \tau^{2}}+\ldots \\
& 4 \pi^{2} \alpha A_{0}^{*} \frac{d^{2} P_{0}^{*}(\xi)}{d \xi^{2}}+4 \pi^{2} \alpha A_{1}^{*} \frac{d^{2} P_{1}^{*}(\xi)}{d \xi^{2}}
\end{aligned}
$$


The method of Bubnov-Galerkin stipulates the above error $\epsilon(\xi, \tau)$ to be orthogonal to each $P_{j}(\xi)$ :

$$
\int_{0}^{1} \epsilon(\xi, \tau) P_{0}^{*}(\xi) d \xi=0, \quad \int_{0}^{1} \epsilon(\xi, \tau) P_{1}^{*}(\xi) d \xi=0
$$

Because of the orthogonality properties of the functions $P_{0}^{*}(\xi)$ and $P_{1}^{*}(\xi)$ and their derivatives $d^{2} P_{0}^{*}(\xi) / d \xi^{2}, d^{2} P_{1}^{*}(\xi) / d \xi^{2}, d^{4} P_{0}^{*}(\xi) / d \xi^{4}$ and $d^{4} P_{1}^{*}(\xi) / d \xi^{4}$ :

$$
\begin{gathered}
\int_{0}^{1} \frac{d^{i} P_{1}^{*}(\xi)}{d \xi^{i}} P_{0}^{*}(\xi)=\int_{0}^{1} \frac{d^{i} P_{0}^{*}(\xi)}{d \xi^{i}} P_{1}^{*}(\xi) d \xi=0 \\
\int_{0}^{1} P_{0}^{*}(\xi) P_{0}^{*}(\xi) d \xi=\frac{3}{2} \quad \int_{0}^{1} \frac{d^{2} P_{0}^{*}(\xi)}{d \xi^{2}} P_{0}^{*}(\xi) d \xi=-2 \pi^{2} \quad \int_{0}^{1} \frac{d^{4} P_{0}^{*}(\xi)}{d \xi^{4}} P_{0}^{*}(\xi)=8 \pi^{4} \\
\int_{0}^{1} P_{1}^{*}(\xi) P_{1}^{*}(\xi) d \xi=1 \quad \int_{0}^{1} \frac{d^{2} P_{1}^{*}(\xi)}{d \xi^{2}} P_{1}^{*}(\xi) d \xi=-5 \pi^{2} \quad \int_{0}^{1} \frac{d^{4} P_{1}^{*}(\xi)}{d \xi^{4}} P_{1}^{*}(\xi)=41 \pi^{4}
\end{gathered}
$$

following relations are obtained:

$$
\begin{gathered}
G_{0}^{*}(\tau)(1-\alpha)+\frac{3}{16} \gamma^{2} \frac{d^{2} G_{0}^{*}(\tau)}{d \tau^{2}}=\alpha A_{0}^{*} \\
G_{1}^{*}(\tau)(41-20 \alpha)+\gamma^{2} \frac{d^{2} G_{1}^{*}(\tau)}{\tau^{2}}=20 \alpha A_{1}^{*}
\end{gathered}
$$

The solution of equation (30) reads $G_{0}^{*}(\tau)=A_{0}^{*} \psi_{0}^{*}(\tau)$ with

$$
\psi_{0}^{*}(\tau)= \begin{cases}\frac{\alpha}{\alpha-1}\left[\cos \left(\frac{4}{\sqrt{3} \gamma} \sqrt{1-\alpha} \tau\right)-1\right] & \text { if } \alpha<1 \\ \frac{16}{3 \gamma^{2}} \frac{\tau^{2}}{2} & \text { if } \alpha=1 \\ \frac{\alpha}{\alpha-1}\left[\cosh \left(\frac{4}{\sqrt{3} \gamma^{2}} \sqrt{\alpha-1} \tau\right)-1\right] & \text { if } \alpha>1\end{cases}
$$


whereas the solution of equation $(31) \operatorname{reads} G_{1}^{*}(\tau)=A_{1}^{*} \psi_{1}^{*}(\tau)$, where

$$
\psi_{1}^{*}(\tau)= \begin{cases}\frac{20 \alpha}{20 \alpha-41}\left[\cos \left(\frac{1}{\gamma} \sqrt{41-20 \alpha} \tau\right)-1\right] & \text { if } \alpha<\frac{41}{20} \\ \frac{41}{\gamma^{2}} \frac{\tau^{2}}{2} & \text { if } \alpha=\frac{41}{20} \\ \frac{20 \alpha}{20 \alpha-41}\left[\cosh \left(\frac{1}{\gamma} \sqrt{20 \alpha-41} \tau\right)-1\right] & \text { if } \alpha>\frac{41}{20}\end{cases}
$$

The total displacement is thus described by:

$$
\nu(\xi, \tau)=u(\xi, \tau)+\eta(\xi)=A_{0}^{*}\left[1+\psi_{0}^{*}(\tau)\right] P_{0}^{*}(\xi)+A_{1}^{*}\left[1+\psi_{1}^{*}(\tau)\right] P_{1}^{*}(\xi)
$$

Hereinafter, the amplitudes $A_{0}$ and $A_{1}$ or $A_{0}^{*}$ and $A_{1}^{*}$ will be either treated as belonging to a convex set or as two random variables. This is done with an express goal of comparing these two philosophically competing methodologies. Now we are interested to find extremal responses when initial imperfections belong to some convex set.

\section{Convex model for the initial imperfection}

Following Ben-Haim and Elishakoff [2], we represent the uncertainty in the initial imperfection profile so that we allow the coefficients $A_{0}$ and $A_{1}$ or $A_{0}^{*}$ and $A_{1}^{*}$ to vary on an ellipse (for simplicity of notation, the analysis hereafter is explicitly written down for $A_{0}$ and $A_{1}$, but these could be easily substituted by $A_{0}^{*}$ and $\left.A_{1}^{*}\right)$ :

$$
Z(\theta, W)=\left\{A=\left(A_{0}, A_{1}\right): A^{T} W A \leq \theta^{2}\right\}
$$

where $W$ is a $2 \times 2$ positive definite real symmetric matrix and $\theta^{2}$ is a positive number. The shape and size of the ellipse are determined by $W$ and $\theta^{2}$, which 
are chosen to represent available information concerning the variability of the coefficients of the initial deflection profile.

Next, the upper bounds on the maximum deflection, duration above a threshold and the integral displacements are calculated.

\subsection{Maximum Deflection}

A widely accepted criterion for failure is the maximum deflection: If the instantaneous displacement exceeds a specified threshold, then failure is presumed to result [9]. The maximum on $Z(\theta, W)$ of the total displacement of the beam at time $\tau$ and position $\xi$ is

$$
\nu_{\max }(\xi, \tau)=\max _{A \in Z(\theta, W)} \nu(\xi, \tau)
$$

Because $\nu(\xi, \tau)$ is a linear function of the coefficients, and because $Z(\theta, W)$ is a convex set, the maximum of $\nu$ occurs on the set $C$ of extreme points of $Z$.

$$
C(\theta, W)=\left\{A=\left(A_{0}, A_{1}\right): A^{T} W A=\theta^{2}\right\}
$$

Thus

$$
\nu_{\max }(\xi, \tau)=\max _{A \in C(\theta, W)} \nu(\xi, \tau)
$$

Let us define a $2 \mathrm{D}$ vector $\phi(\xi, \tau)$ with elements

$$
\phi_{0}(\xi, \tau)=\left(1+\psi_{0}(\tau)\right) P_{0}(\xi), \quad \phi_{1}(\xi, \tau)=\left(1+\psi_{1}(\tau)\right) P_{1}(\xi)
$$

We wish to maximize $A^{T} \phi(\xi, \tau)$ subject to the constraint that A falls on the extreme points of $Z$. Let the Lagragian be

$$
H=A^{T} \phi(\xi, \tau)+\lambda\left(A^{T} W A-\theta^{2}\right)
$$


A necessary condition for an extremum is

$$
0=\frac{\partial H}{\partial A}=\phi(\xi, \tau)+2 \lambda W A \rightarrow A=\frac{1}{2 \lambda}-W^{-1} \phi(\xi, \tau)
$$

Combining this equation with equation (37), one finds

$$
\lambda^{2}=\frac{1}{4 \theta^{2}} \phi(\xi, \tau)^{T} W^{-1} \phi(\xi, \tau)
$$

so that

$$
A=\frac{\theta}{\sqrt{\phi(\xi, \tau)^{T} W^{-1} \phi(\xi, \tau)}} W^{-1} \phi(\xi, \tau)
$$

and the maximum total displacement of the beam, at position $\xi$ and time $\tau$, is

$$
\nu_{\max }(\xi, \tau)=\theta \sqrt{\phi(\xi, \tau)^{T} W^{-1} \phi(\xi, \tau)}
$$

Let us consider a numerical example to shed some light on the failure of the clamped-clamped beam. The set of allowed amplitudes $A=\left(A_{0}, A_{1}\right)$ is represented as a circle centered at the origin (so that $W$ is the $2 \times 2$ identity matrix), with radius $\theta=0.07$. To allow for a fair comparison of both solution methods the initial imperfection uncertainty for a set of specified points along the beam should be the same in both approaches. We choose to take the same value for $\theta$ and consider the diagonal structure of $W$. Since two uncertain amplitudes are involved, we can specify two locations along the beam for which the initial imperfection uncertainty should be the same in both solutions. For example, we want to allow the imperfection at the mid-point of the beam and at one quarter of the length of the beam to vary in the same interval as in the exact mode shape representation. Substituting in equation 44 the values for the exact mode solution one finds $\nu_{\max }(0.5,0)=0.111$ and $\nu_{\max }(0.25,0)=0.118$. 
Equating these to

$$
\begin{aligned}
& 0.111=\theta \sqrt{\phi_{0}^{* 2}(0.5,0) W_{11}^{*-1}+\phi_{1}^{* 2}(0.5,0) W_{22}^{*-1}}=\theta \sqrt{\phi_{0}^{* 2}(0.5,0) W_{11}^{*-1}} \\
& 0.118=\theta \sqrt{\phi_{0}^{* 2}(0.25,0) W_{11}^{*-1}+\phi_{1}^{* 2}(0.25,0) W_{22}^{*-1}}
\end{aligned}
$$

we find that the allowed amplitudes $A^{*}=\left(A_{0}^{*}, A_{1}^{*}\right)$ should belong to the ellips $A^{* T} W^{*} A^{*} \leq \theta^{2}$ with

$$
W^{*}=\left[\begin{array}{cc}
1.586 & 0 \\
0 & 0.9088
\end{array}\right]
$$

and $\theta=0.07$. Indeed, the initial imperfection uncertainty at the mid-point of the beam is in both descriptions equal to the interval $[-0.111,0.111]$ and at one quarter of the length of the beam this is $[-0.118,0.118]$. The value for $\gamma$ in the expression for the first natural frequency $\omega_{1}$ is 2.27 . The value for $\zeta$ in the expression for the second natural frequency is 6.25 . In the normal mode solution following constants are taken $\kappa=4.99$ and $\eta=18.66$.

Figure 2 shows the maximum total deflection at two points on the beam $(\xi=0.25$ and $\xi=0.5)$ as a function of time. The axial load ratio is $\alpha=3$. The maximum possible midpoint deflection is lower than the deflection at 0.25 times the length. This is due to the loading which triggers a buckling pattern with a higher emphasis on the second normal mode or second term in the F-B series. Furthermore, the normal mode solution appears to give a lower value for the maximum deflection for all shown time instances at both locations $\xi=0.25$ and $\xi=0.5$.

Figure 3 shows the maximum normalized deflection at $\xi=0.25$ as a function of time, for several values of the axial load ratio. The maximum deflection increases sharply as the axial load is increased. The conclusion here is that the least favorable displacement can be evaluated for any specified value of the load. The normal mode solution appears to give a lower value for the maximum 


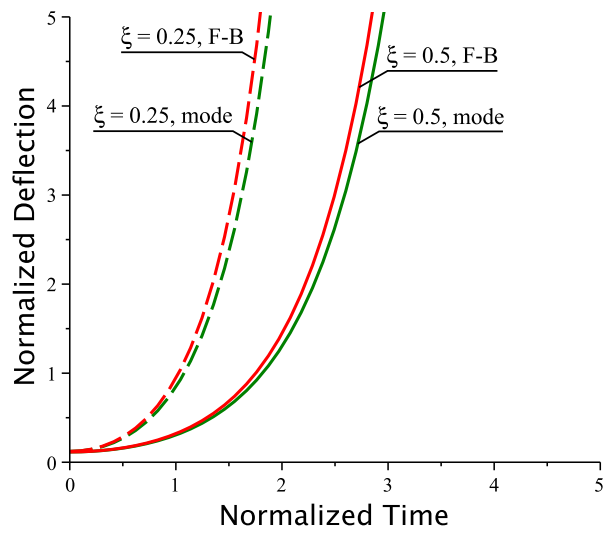

Figure 2: Maximum normalized deflection versus normalized time at $\xi=0.25$ (dashed line) and $\xi=0.5$ (solid line). Load ratio $\alpha=3$. The green lines correspond to the solution with the normal modes; the red lines correspond to the solution with two term Filonenko-Borodich (F-B) approximation

deflection for all shown time instances and all three calculated load ratios.

\subsection{Maximum Integral Displacements}

We consider the integral (span-averaged) displacement along the beam at time $\tau$

$$
\begin{gathered}
\delta(\tau)=\int_{0}^{1} \nu(\xi, \tau) d \xi=\beta A_{0}\left[1+\psi_{0}(\tau)\right] \\
\delta^{*}(\tau)=\int_{0}^{1} \nu^{*}(\xi, \tau) d \xi=A_{0}^{*}\left[1+\psi_{0}^{*}(\tau)\right]
\end{gathered}
$$

since the integral of $P_{0}(\xi)$ and $P_{1}(\xi)$ over the length of the beam are equal to $\beta=0.831$ and zero, respectively. Furthermore, the integral of $P_{0}^{*}(\xi)$ and $P_{1}^{*}(\xi)$ over the length of the beam are equal to one and zero, respectively.

To calculate the maximum integral deflection for the requirement that $A$ belongs to $Z(\theta, W)$, the same procedure as in section 4.1 is followed. Where the $2 D$-vector $\phi(\xi, \tau)$ is now alternatively defined as $\phi(\tau)$ :

$$
\phi_{0}(\tau)=1+\psi_{0}(\tau), \quad \phi_{1}(\tau)=0
$$




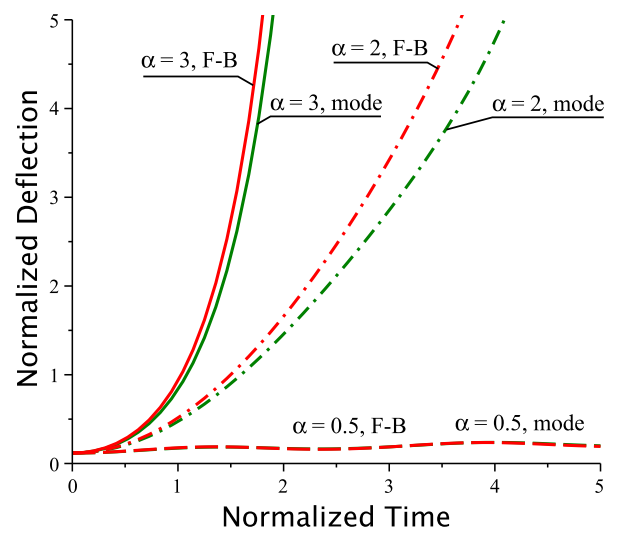

Figure 3: Maximum normalized deflection versus normalized time at $\xi=0.25$ for various values of the load ratio. The green lines correspond to the solution with the normal modes; the red lines correspond to the solution with two term Filonenko-Borodich (F-B) approximation.

The procedure formally yields the same expression as in eq. (44) but now expressed for the maximum of $\delta\left(\right.$ or $\left.\delta^{*}\right)$ :

$$
\delta_{\max }(\tau)=\theta \sqrt{\phi(\tau)^{T} W^{-1} \phi(\tau)}
$$

Figure 4 shows the maximum span-averaged displacement versus the normalized time, for the load ratio $\alpha=0.5$ and $\alpha=3$. For this measure, the solution with the normal mode and the one term Filonenko-Borodich approximation appear to be very close to each other. This observation indicates that the difference between the approximate solution and the exact mode shape solution after spatial integration becomes very small.

\section{Probabilistic model for the initial imperfections}

We represent the uncertainty in the initial imperfection profile so that we treat the coefficients $A_{0}$ and $A_{1}$ as random variables [5] with following uniform 


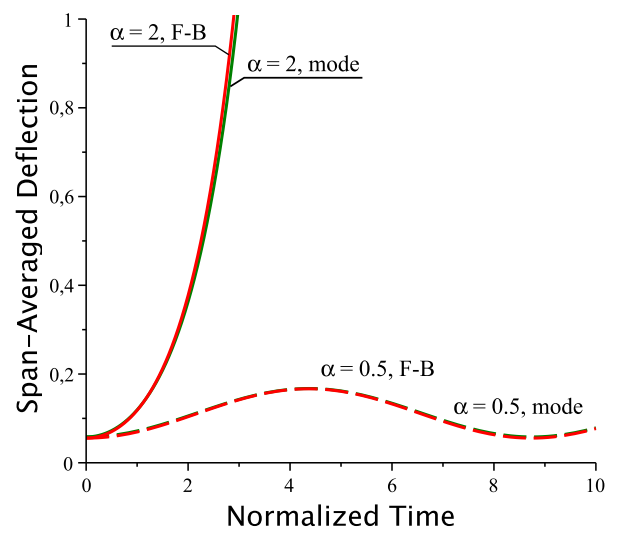

Figure 4: Maximum integral (span-averaged) deflection versus normalized time for various values of the load ratio. The green lines correspond to the solution with the normal modes; the red lines correspond to the solution with two term Filonenko-Borodich (F-B) approximation.

joint probability density function (pdf):

$$
f_{A_{0}, A_{1}}= \begin{cases}\frac{1}{\pi r^{2}} & \text { if } \sqrt{A_{0}^{2}+A_{1}^{2}} \leq r \\ 0 & \text { otherwise }\end{cases}
$$

or equivalently a uniform distribution $f_{A_{0}^{*}, A_{1}^{*}}^{*}$ over an elliptic area

$$
f_{A_{0}^{*}, A_{1}^{*}}^{*}= \begin{cases}\frac{1}{\pi d e} & \text { if } \sqrt{A_{0}^{* 2} / d+A_{1}^{* 2} / e} \leq r \\ 0 & \text { otherwise }\end{cases}
$$

(For simplicity of notation, the analysis hereinafter is explicitly written down for $A_{0}$ and $A_{1}$, but these could be straightforwardly substituted by $A_{0}^{*}$ and $A_{1}^{*}$.) The interested reader can find the analysis with a truncated normal distribution in Appendix.

The failure is identified with the reaching by the absolute value of the total displacements the critical point $c$. We are interested in the reliability $R=$ $\operatorname{Prob}(\Lambda \geq \lambda)$ of the first excursion time $\Lambda$ being equal to or greater than the 
given time $\lambda$. We now proceed to calculate $R(\lambda)$, in accordance with equation (34):

$$
\begin{aligned}
R(\lambda)= & \operatorname{Prob}[|\nu(\xi, \lambda)| \leq c] \\
= & \operatorname{Prob}\left\{\left|A_{0}\left[1+\psi_{0}(\lambda)\right] P_{0}(\xi)+A_{1}\left[1+\psi_{1}(\lambda)\right] P_{1}(\xi)\right| \leq c\right\} \\
= & \operatorname{Prob}\left[\left\{A_{0}\left[1+\psi_{0}(\lambda)\right] P_{0}(\xi)+A_{1}\left[1+\psi_{1}(\lambda)\right] P_{1}(\xi) \geq-c\right\} \cap\right. \\
& \left.\left\{A_{0}\left[1+\psi_{0}(\lambda)\right] P_{0}(\xi)+A_{1}\left[1+\psi_{1}(\lambda)\right] P_{1}(\xi) \leq c\right\}\right]
\end{aligned}
$$

In general the resulting reliability will be calculated by integrating the probability density over an area as shown in Figure 5 . It is clear from the figure that

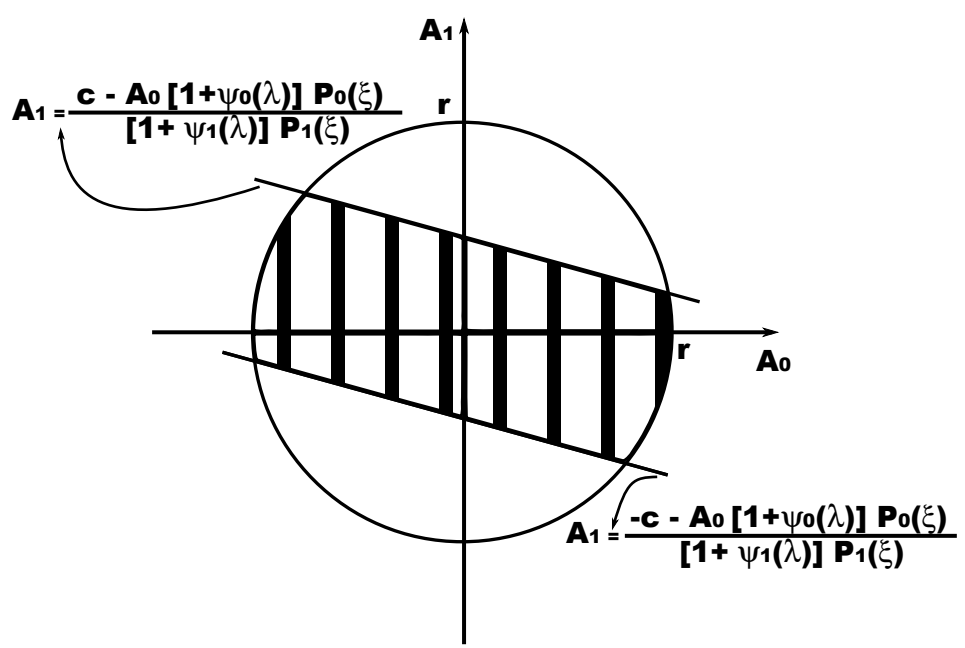

Figure 5: The calculation of the reliability is done by integrating the probability density function over an area similar to the one shaded in the figure.

the particular value for the allowable deflection $c$, the spatial location along the beam $\xi$ and the time of interest $\lambda$ will influence the area of integration and thus the reliability. Naturally, the load ratio $\alpha$ is a parameter in $\psi(\lambda)$ and will also influence the reliability.

Let us consider a numerical example. As an example, let us consider a 
pdf for $\left(A_{0}, A_{1}\right)$ as in Eq. (52) with $r=0.07$ and for $\left(A_{0}^{*}, A_{1}^{*}\right)$ a pdf as in Eq. (53) with $d=0.631, e=1.1, r=0.07$ so that the bounds on the initial imperfection at $\xi=0.25$ are the same in both solution methods. We investigate the reliability for $\xi=0.25$ in Eq. (54). The applied load is considered to be half, two and three times the classical buckling load ( $\alpha=0.5,2$ and 3 , respectively). The reliability is depicted in figures 6,7 and 8 for the cases where the ciritical point $c=0.13$ (dashed lines) and $c=0.15$ (solid lines) for $\alpha=0.5,2$ and 3, respectively. It is clear from the three pictures that with increasing load ratio the reliability is decreasing, as it should be. Furthermore, for all calculated cases the approximate solution assigns a lower reliability to the beam when compared to the exact mode shape solution. This means that in this particular analysis, designing according to the approximate solution method would result in a conservative design. Naturally, this unfortunately does not hold in general.

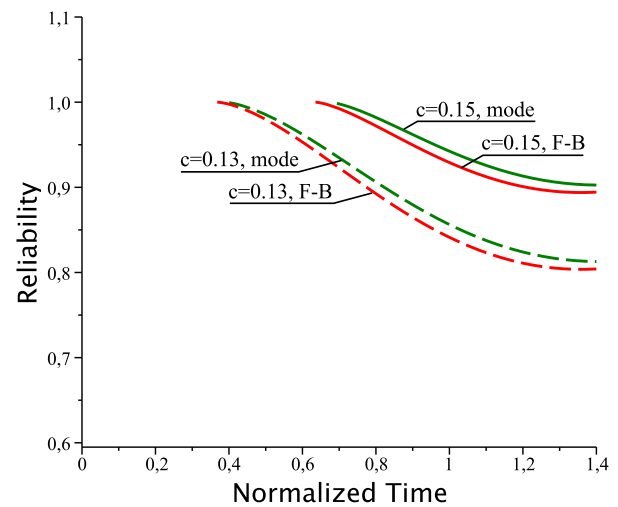

Figure 6: The reliability as a function of time $(\alpha=0.5)$. The green lines correspond to the solution with the normal modes; the red lines correspond to the solution with two term Filonenko-Borodich (F-B) approximation. 


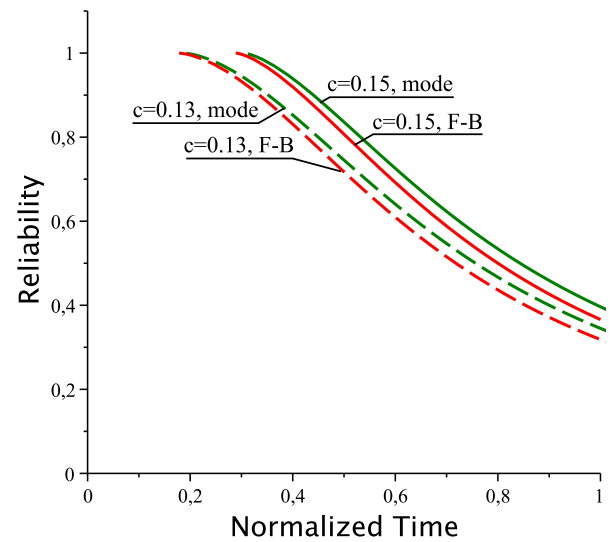

Figure 7: The reliability as a function of time $(\alpha=2)$. The green lines correspond to the solution with the normal modes; the red lines correspond to the solution with two term Filonenko-Borodich (F-B) approximation.

\begin{tabular}{|r|c|} 
Reliability & Design Time $\tau$ \\
\hline 0.9 & 1.4 \\
0.95 & 0.95 \\
0.99 & 0.75 \\
0.995 & 0.72 \\
0.999 & 0.70 \\
1 & 0.69
\end{tabular}

Table 1: The design time for a clamped beam under axial impact loading for different levels of the required reliability based on the exact mode solution

\section{Comparison of Probabilistic and Convex Analyses}

The comparison between the probabilistic and convex analyses consists in comparing the value assigned to a design variable by the convex model and the probabilistic model. Let us consider the design time $\tau$ of the clamped beam under the axial impact load for increasing levels of the reliability. Furthermore, let us first concentrate on the exact mode solution for a load ratio $\alpha=0.5$ and critical value $c=0.15$. Table 1 shows the values for the design time for increasing values of the demanded reliability for the considered case. These values can be directly derived from Figure 6. From the convex analysis, we can derive the first time the maximum possible deflection of the point $\xi=0.25$ 


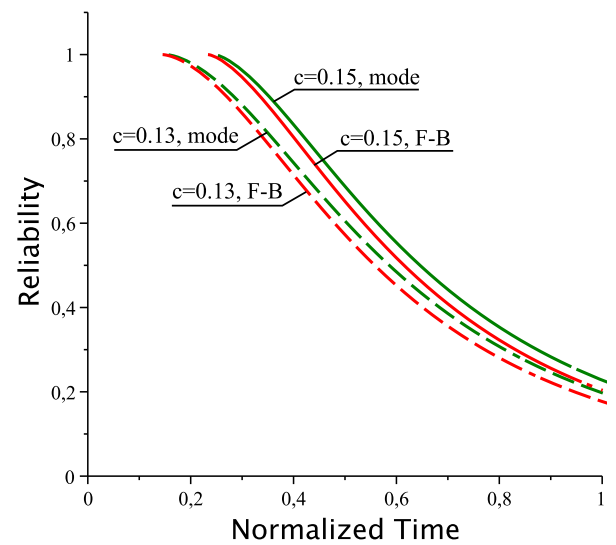

Figure 8: The reliability as a function of time $(\alpha=3)$. The green lines correspond to the solution with the normal modes; the red lines correspond to the solution with two term Filonenko-Borodich (F-B) approximation.

can surpass the critical value $c=0.15$. From Figure 3 and Equation (44) it is confirmed that the first passage time is 0.69 . This value coincides with the fail-safe life $\tau_{0}$ for a reliability tending to one as found above. Indeed, for a reliability tending to one from below, the area of integration consists of the entire circle since the limiting curves (representing the boundary between safe operation and failure) intersect the circumference of the circle at a single point only, as depicted in Figure 9.

Next, let us consider the approximative solution using the Filonenko-Borodich "cosinusoidal polynomials" for the exact same case. The value for the design time as a function of the demanded reliability is shown in Table 2. The fail-safe life turns out to be $\tau_{0}^{*}=0.64$ By setting the ${ }^{*}$ equivalent of Equation (44) equal to 0.15 and solving for $\lambda$, we find the first passage time to be $\tau_{0}^{*}=0.64$. Again, the convex model and the probabilistic model agree on the fail-safe life. The same conclusion can be drawn for the comparison between the convex model and the probabilistic model with a truncated normal distribution as shown in the Appendix. 


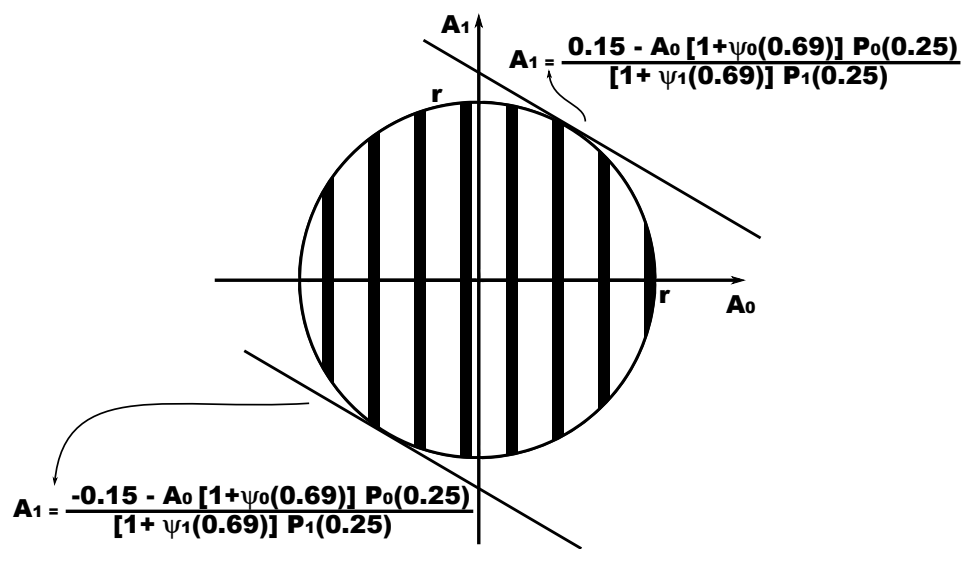

Figure 9: The area of integration of the probability density function for the reliability tending to one from below.

\begin{tabular}{|r|c|} 
Reliability & Design Time $\tau^{*}$ \\
\hline 0.9 & 1.2 \\
0.95 & 0.89 \\
0.99 & 0.71 \\
0.995 & 0.68 \\
0.999 & 0.66 \\
0.9995 & 0.65 \\
1 & 0.64
\end{tabular}

Table 2: The design time for a clamped beam under axial impact loading for different levels of the required reliability based on the approximate solution using Filonenko-Borodich "cosinusoidal polynomials" 
What can be said about the preference for either uncertainty description (probabilistic or convex model) based on this coinciding result? Our answer should be balanced. If a design engineer is interested to find a fail-safe life, one does not need the probabilistic description of the uncertainties. In general, for reliabilities tending to one, the probabilistic details captured in a pdf are hidden and only the limiting values on an uncertainty are apparent in the outcome. The design strategy which requires the reliability approaching unity actually converges to a worst case design strategy as pursued by the convex analysis. In this case one can follow the principle of Ockham's Razor: "What can be done with fewer [assumptions] is done in vain with more." On the other hand, if the same design engineer is conducting analyses for reliabilities lower than one, more information than only the limiting bounds on the uncertainty is needed. In such analyses, one has to have sufficient proofs for the validity of the applied pdf and the values adopted for its parameters to come up with reliable reliability calculations. To summarize, both methods, the convex and probabilistic one can live in so called peaceful coexistence as they each have their strengths in similar but seperate domains.

\section{Conclusions}

The paper presents the analysis of a clamped beam with initial imperfections under impact buckling. A solution method based on the exact mode shapes of a clamped beam and an approximate solution method based on the FilonenkoBorodich series are carried out. The focus is on the treatment of the uncertainty in the initial imperfections and its consequences for the output. It is shown that the calculation of the design time for fail-safe life does not require a full probabilistic analysis. If insufficient probabilistic information is available, it is unnecessary and inefficient to adopt arbitrary distribution functions for the 
imperfection amplitudes. The convex analysis delivers the design time without additional assumptions in a straightforward analytical form. This holds for both the exact mode and approximative solution that are studied. The study confirms the well-known equivalence between convex and probabilistic analyses for reliability tending to one in the solution of an impact buckling problem of a beam with clamped boundary conditions.

\section{Acknowledgments}

This study was performed during the research stay of W.V. at the Florida Atlantic University. W.V. would like to acknowledge the highly appreciated support of the IWT-Flanders, the Commission for Educational Exchange Between The United States of America, Belgium and Luxembourg (The Fulbright Fund and Vesuvius) and the Raymond Snoeys fund of the KU Leuven. The authors would like to thank the reviewers for their valuable comments.

\section{Appendix}

Probabilistic analysis with truncated normal distribution

We represent the uncertainty in the initial imperfection profile so that we treat the coefficients $A_{0}$ and $A_{1}$ as random variables [5] with following truncated normal joint probability density function (pdf):

$$
f_{A_{0}, A_{1}}= \begin{cases}\frac{1}{2 M \pi \sigma_{A_{0}} \sigma_{A_{1}}} e^{-\frac{1}{2}\left(\frac{A_{0}^{2}}{\sigma_{A_{0}}^{2}}+\frac{A_{1}^{2}}{\sigma_{A_{1}}^{2}}\right)} & \text { if } \sqrt{A_{0}^{2}+A_{1}^{2}} \leq r \\ 0 & \text { otherwise }\end{cases}
$$


or equivalently a truncated normal distribution $f_{A_{0}^{*}, A_{1}^{*}}^{*}$ over an elliptic area

$$
f_{A_{0}^{*}, A_{1}^{*}}^{*}= \begin{cases}\frac{1}{2 N \pi \sigma_{A_{0}^{*}} \sigma_{A_{1}^{*}}} e^{-\frac{1}{2}\left(\frac{A_{0}^{* 2}}{\sigma_{A_{0}^{*}}^{2}}+\frac{A_{1}^{* 2}}{\sigma_{A_{1}^{*}}^{2}}\right)} & \text { if } \sqrt{A_{0}^{* 2} / d+A_{1}^{* 2} / e} \leq r \\ 0 & \text { otherwise }\end{cases}
$$

with

$$
M=4 \int_{0}^{r} \int_{0}^{\sqrt{r^{2}-A_{0}^{2}}} \frac{1}{2 \pi \sigma_{A_{0}} \sigma_{A_{1}}} e^{-\frac{1}{2}\left(\frac{A_{0}^{2}}{\sigma_{A_{0}}^{2}}+\frac{A_{1}^{2}}{\sigma_{A_{1}}^{2}}\right)} \mathrm{d} A_{1} \mathrm{~d} A_{0}
$$

the integral of the normal probability density function over the circular region and

$$
N=4 \int_{0}^{r / d} \int_{0}^{\sqrt{\frac{r^{2}-d^{2} A_{0}^{* 2}}{e^{2}}}} \frac{1}{2 \pi \sigma_{A_{0}^{*}} \sigma_{A_{1}^{*}}} e^{-\frac{1}{2}\left(\frac{A_{0}^{* 2}}{\sigma_{A_{0}^{*}}^{2}}+\frac{A_{1}^{* 2}}{\sigma_{A_{1}^{*}}^{2}}\right)} \mathrm{d} A_{1}^{* 2} \mathrm{~d} A_{0}^{* 2}
$$

the integral of the normal probability density function over the elliptical region. (For simplicity of notation, the analysis hereinafter is explicitly written down for $A_{0}$ and $A_{1}$, but these could be straightforwardly substituted by $A_{0}^{*}$ and $A_{1}^{*}$.)

The failure probability is calculated as before using Equation (54) and the reliability can then be calculated as illustrated in Figure 5.

For the same numerical data as in the main text, but with the uniform density replaced by the above described truncated normal distribution with $\sigma_{A_{0}}=\sigma_{A_{1}}=\sigma_{A_{0}^{*}}=\sigma_{A_{1}^{*}}=0.05$, the reliability is depicted in figures 10, 11 and 12 for the cases where the ciritical point $c=0.13$ (dashed lines) and $c=0.15$ (solid lines) for $\alpha=0.5,2$ and 3, respectively. The same conclusions hold: it is clear from the three pictures that with increasing load ratio the reliability is decreasing, as it should be. Furthermore, for all calculated cases the approximate solution assigns a lower reliability to the beam when compared to the exact mode shape solution. This means that in this particular analysis, designing according to the approximate solution method would result in a conservative design. Naturally, this unfortunately does not hold in general. 


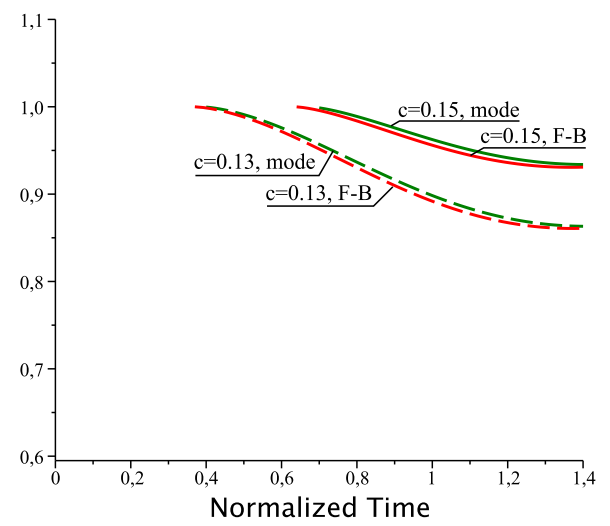

Figure 10: The reliability as a function of time $(\alpha=0.5)$. The green lines correspond to the solution with the normal modes; the red lines correspond to the solution with two term Filonenko-Borodich (F-B) approximation.

\begin{tabular}{|r|c|} 
Reliability & Design Time $\tau$ \\
\hline 0.93 & 1.4 \\
0.95 & 1.1 \\
0.97 & 0.95 \\
0.99 & 0.77 \\
0.995 & 0.74 \\
0.999 & 0.70 \\
1 & 0.69
\end{tabular}

Table 3: The design time for a clamped beam under axial impact loading for different levels of the required reliability based on the exact mode solution

\section{Comparison of Probabilistic and Convex Analyses}

The comparison between the probabilistic and convex analyses follows the same procedure as in the main text. Table 3 shows the values for the design time for increasing values of the demanded reliability for the considered case. These values can be directly derived from Figure 10. In comparison with the values of table 1 that are based on the uniform density, it is clear that this reliability is a slower decreasing function of the design time. This is a result of the density towards the boundary of the circular domain being less than the uniform value. On the other hand, since most of the density is concentrated 


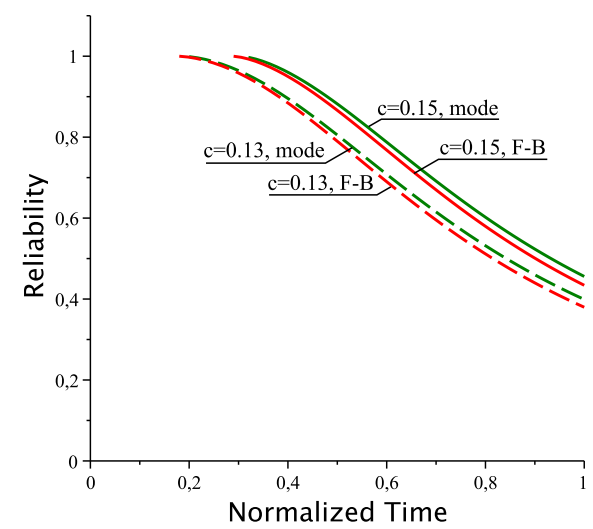

Figure 11: The reliability as a function of time $(\alpha=2)$. The green lines correspond to the solution with the normal modes; the red lines correspond to the solution with two term Filonenko-Borodich (F-B) approximation.

around the middle of the circular domain and thus already accounted for when calculating the high reliability design times this results in higher reliabilities for the same design times when compared with 1 . However, the important feature of the two types of densities is their boundedness on the same domain and thus they agree on the design time for reliability equal to one. Furthermore, they agree with the convex analysis result as found in the main text.

Next, let us consider the approximative solution using the Filonenko-Borodich "cosinusoidal polynomials" for the exact same case. The value for the design time as a function of the demanded reliability is shown in Table 4. The fail-safe life turns out to be $\tau_{0}^{*}=0.64$. The same conclusions regarding the agreement between the probabilistic models for reliability tending to one and the convex model hold here too.

\section{References}

[1] D. Anderson and H. Lindberg. Dynamic pulse buckling of cylindrical shells under transient lateral pressures. AIAA Journal, 6(4):589-598, 1968. 


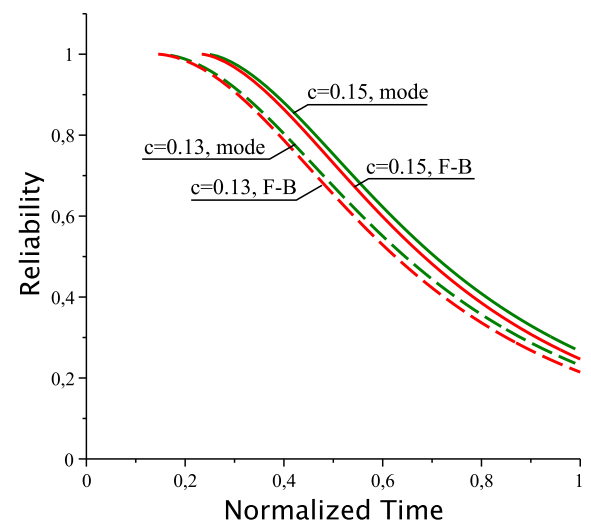

Figure 12: The reliability as a function of time $(\alpha=3)$. The green lines correspond to the solution with the normal modes; the red lines correspond to the solution with two term Filonenko-Borodich (F-B) approximation.

\begin{tabular}{|r|c|} 
Reliability & Design Time $\tau^{*}$ \\
\hline 0.93 & 1.3 \\
0.95 & 1.05 \\
0.99 & 0.75 \\
0.995 & 0.71 \\
0.999 & 0.66 \\
0.9995 & 0.65 \\
1 & 0.64
\end{tabular}

Table 4: The design time for a clamped beam under axial impact loading for different levels of the required reliability based on the approximate solution using Filonenko-Borodich "cosinusoidal polynomials"

[2] Y. Ben-Haim and I. Elishakoff. Convex Models of Uncertainty in Applied Mechanics. Elsevier, 1990.

[3] D. Blackketter and L. May. Permanent deformation of an axial rod subjected to dynamic loading. AIAA Journal, 7(8):1651-1653, 1969.

[4] D. Danielson. Dynamic buckling loads of imperfection-sensitive structures from perturbation procedures. AIAA Journal, 7(8):1506-1510, 1969.

[5] I. Elishakoff. Axial impact buckling of a column with random initial im- 
perfections. Journal of Applied Mechanics, 45(2):361-365, 1978.

[6] I. Elishakoff. Impact buckling of thin bar via monte carlo method. Journal of Applied Mechanics, 45(3):586-590, 1978.

[7] M.M. Filonenko-Borodich. On a certain system of functions and its applications in theory of elasticity. Prikladnaia Matematika i Mekhanika (Journal of Applied Mathematics and Mechanics), 10:193-208 (in Russian), 1946.

[8] J. Garrelick and J. Benveniste. Comparison of beam impact models. AIAA Journal, 8(4):823-825, 1970.

[9] N.J. Hoff. The dynamics of the buckling of elastic columns. ASME Journal of Applied Mechanics, 18:68-71, 1951.

[10] J. Hu and Z. Qiu. Non-probabilistic convex models and interval analysis method for dynamic response of a beam with bounded uncertainty. Applied Mathematical Modelling, 34(3):725-734, 2010.

[11] J. Hutchinson and B. Budiansky. Dynamic buckling estimates. AIAA Journal, 4(3):525-530, 1966.

[12] A. Kounadis. Nonlinear dynamic buckling of discrete dissipative or nondissipative systems under step loading. AIAA Journal, 29(2):280-289, 1991.

[13] H.E. Lindberg. Impact buckling of a thin bar. ASME Journal of Applied Mechanics, 32:312-322, 1965.

[14] H.E. Lindberg. Dynamic response and buckling failure measures for structures with bounded and random imperfections. Journal of Applied Mechanics, 58(4):1092-1095, 1991.

[15] H.E. Lindberg and A.L. Florence. Dynamic pulse buckling: theory and experiment. Martinus Nijhoff Publishers, Dordrecht, 1987. 
[16] Z. Qiu and X. Wang. Interval analysis method and convex models for impulsive response of structures with uncertain-but-bounded external loads. Acta Mechanica Sinica, 22(3):265-276, 2006.

[17] S. Rao. Vibration of continuous systems. Wiley, New York, 2007.

[18] N. Vilenkin. On some nearly periodic systems of functions. Prikladnaia Matematika i Mekhanika (Journal of Applied Mathematics and Mechanics), 16(3):812-814 (in Russian), 1952.

[19] X. Wang, I. Elishakoff, Z. Qiu, and L. Ma. Comparisons of probabilistic and two nonprobabilistic methods for uncertain imperfection sensitivity of a column on a nonlinear mixed quadratic-cubic foundation. Journal of Applied Mechanics, 76:011007-1, 2009. 


\begin{tabular}{|r|c|} 
Reliability & Design Time $\tau$ \\
\hline 0.9 & 1.4 \\
0.95 & 0.95 \\
0.99 & 0.75 \\
0.995 & 0.72 \\
0.999 & 0.70 \\
1 & 0.69
\end{tabular}

Table 1: The design time for a clamped beam under axial impact loading for different levels of the required reliability based on the exact mode solution

\begin{tabular}{|r|c|} 
Reliability & Design Time $\tau^{*}$ \\
\hline 0.9 & 1.2 \\
0.95 & 0.89 \\
0.99 & 0.71 \\
0.995 & 0.68 \\
0.999 & 0.66 \\
0.9995 & 0.65 \\
1 & 0.64
\end{tabular}

Table 2: The design time for a clamped beam under axial impact loading for different levels of the required reliability based on the approximate solution using Filonenko-Borodich "cosinusoidal polynomials" 


\begin{tabular}{|r|c|} 
Reliability & Design Time $\tau$ \\
\hline 0.93 & 1.4 \\
0.95 & 1.1 \\
0.97 & 0.95 \\
0.99 & 0.77 \\
0.995 & 0.74 \\
0.999 & 0.70 \\
1 & 0.69
\end{tabular}

Table 3: The design time for a clamped beam under axial impact loading for different levels of the required reliability based on the exact mode solution

\begin{tabular}{|r|c|} 
Reliability & Design Time $\tau^{*}$ \\
\hline 0.93 & 1.3 \\
0.95 & 1.05 \\
0.99 & 0.75 \\
0.995 & 0.71 \\
0.999 & 0.66 \\
0.9995 & 0.65 \\
1 & 0.64
\end{tabular}

Table 4: The design time for a clamped beam under axial impact loading for different levels of the required reliability based on the approximate solution using Filonenko-Borodich "cosinusoidal polynomials" 


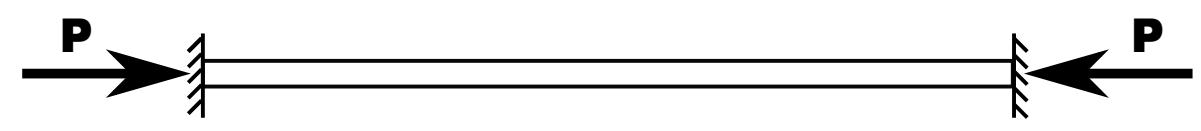

Figure 1: The bi-clamped beam with axial impact loading. 


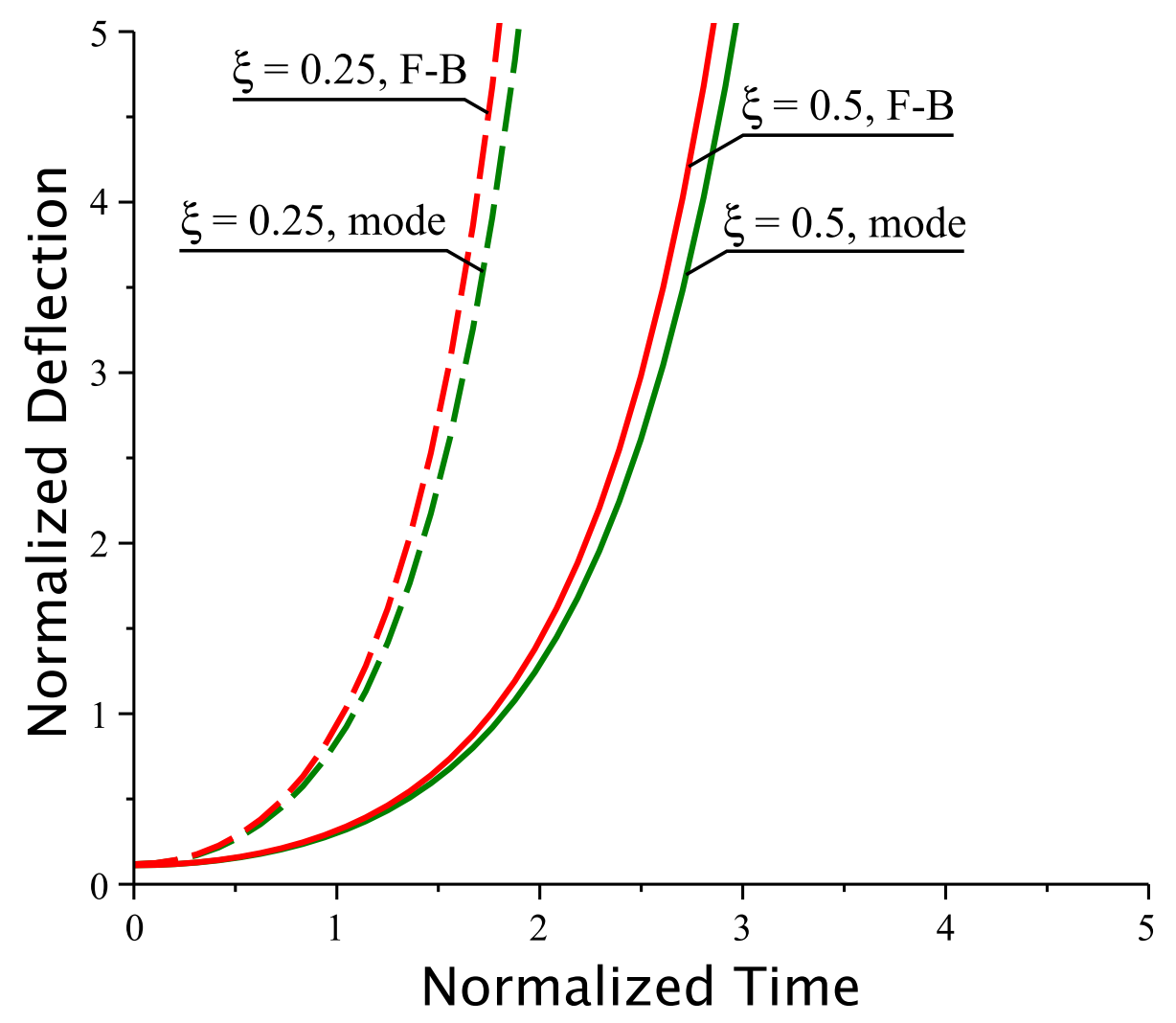

Figure 2: Maximum normalized deflection versus normalized time at $\xi=0.25$ (dashed line) and $\xi=0.5$ (solid line). Load ratio $\alpha=3$. The green lines correspond to the solution with the normal modes; the red lines correspond to the solution with two term Filonenko-Borodich (F-B) approximation 


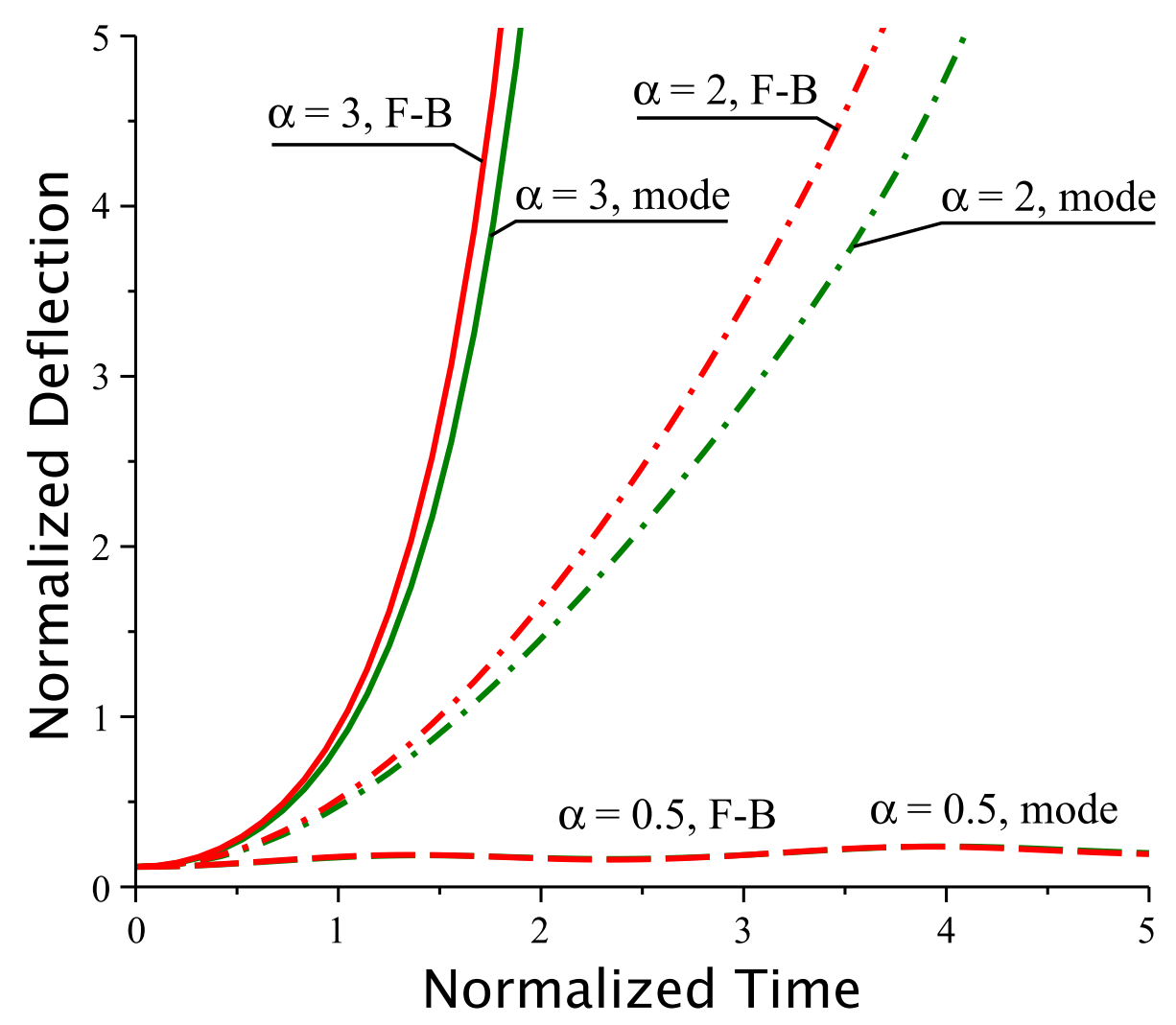

Figure 3: Maximum normalized deflection versus normalized time at $\xi=0.25$ for various values of the load ratio. The green lines correspond to the solution with the normal modes; the red lines correspond to the solution with two term Filonenko-Borodich (F-B) approximation 


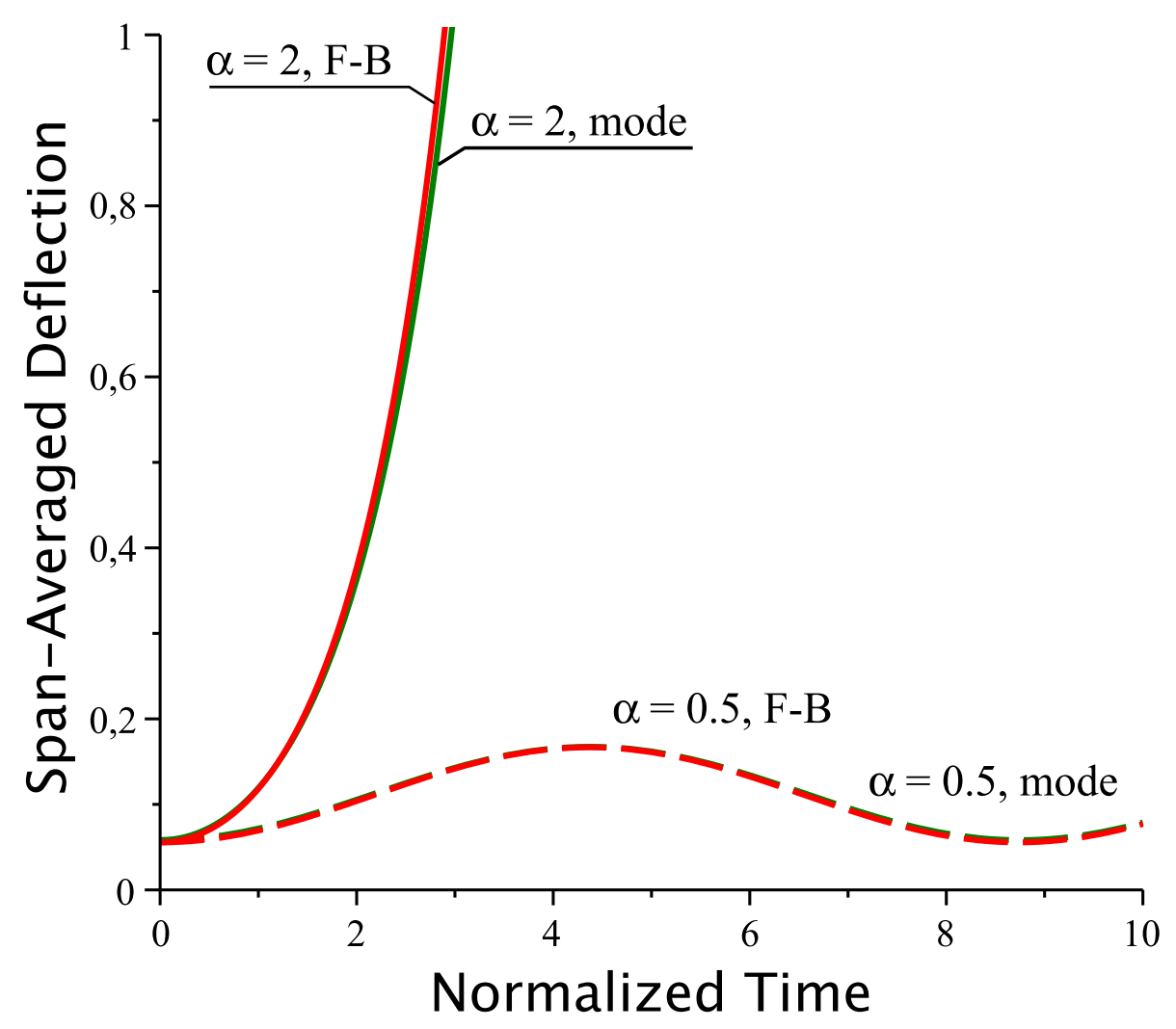

Figure 4: Maximum integral (span-averaged) deflection versus normalized time for various values of the load ratio. The green lines correspond to the solution with the normal modes; the red lines correspond to the solution with two term Filonenko-Borodich (F-B) approximation. 


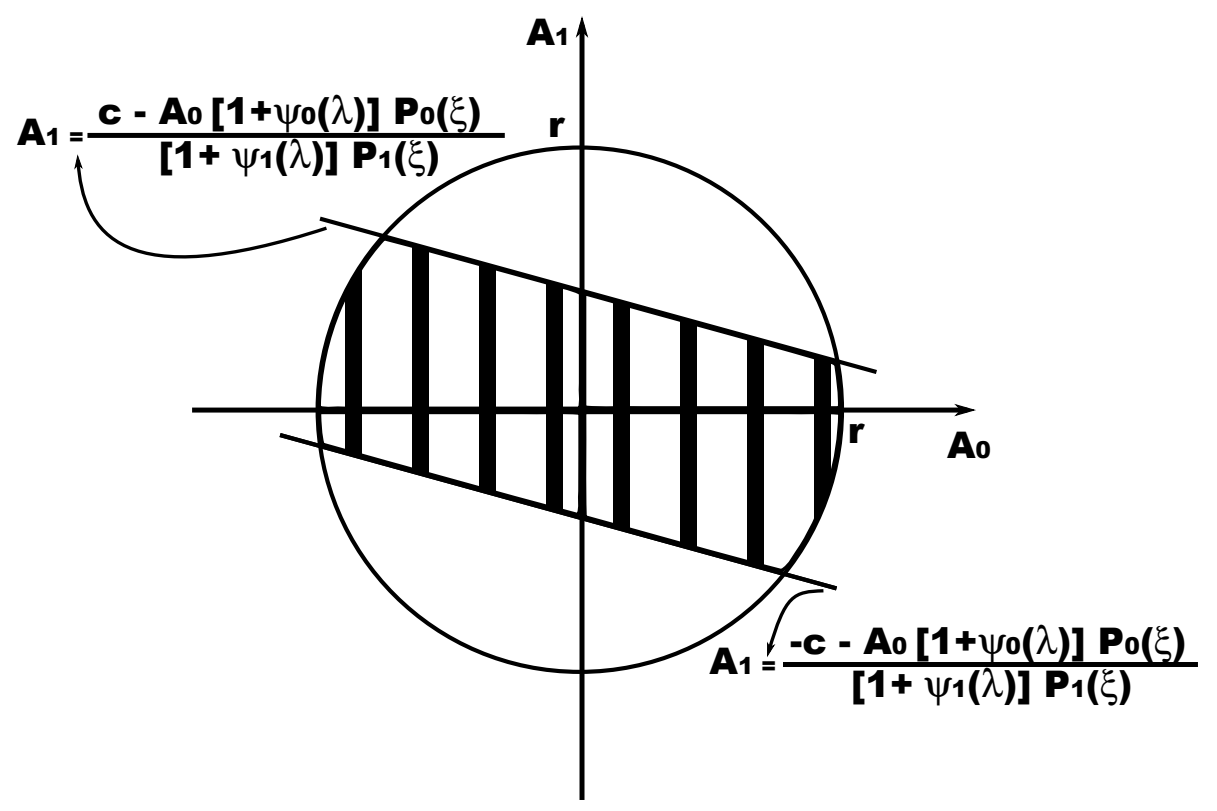

Figure 5: The calculation of the reliability is done by integrating the probability density function over an area similar to the one shaded in the figure. 


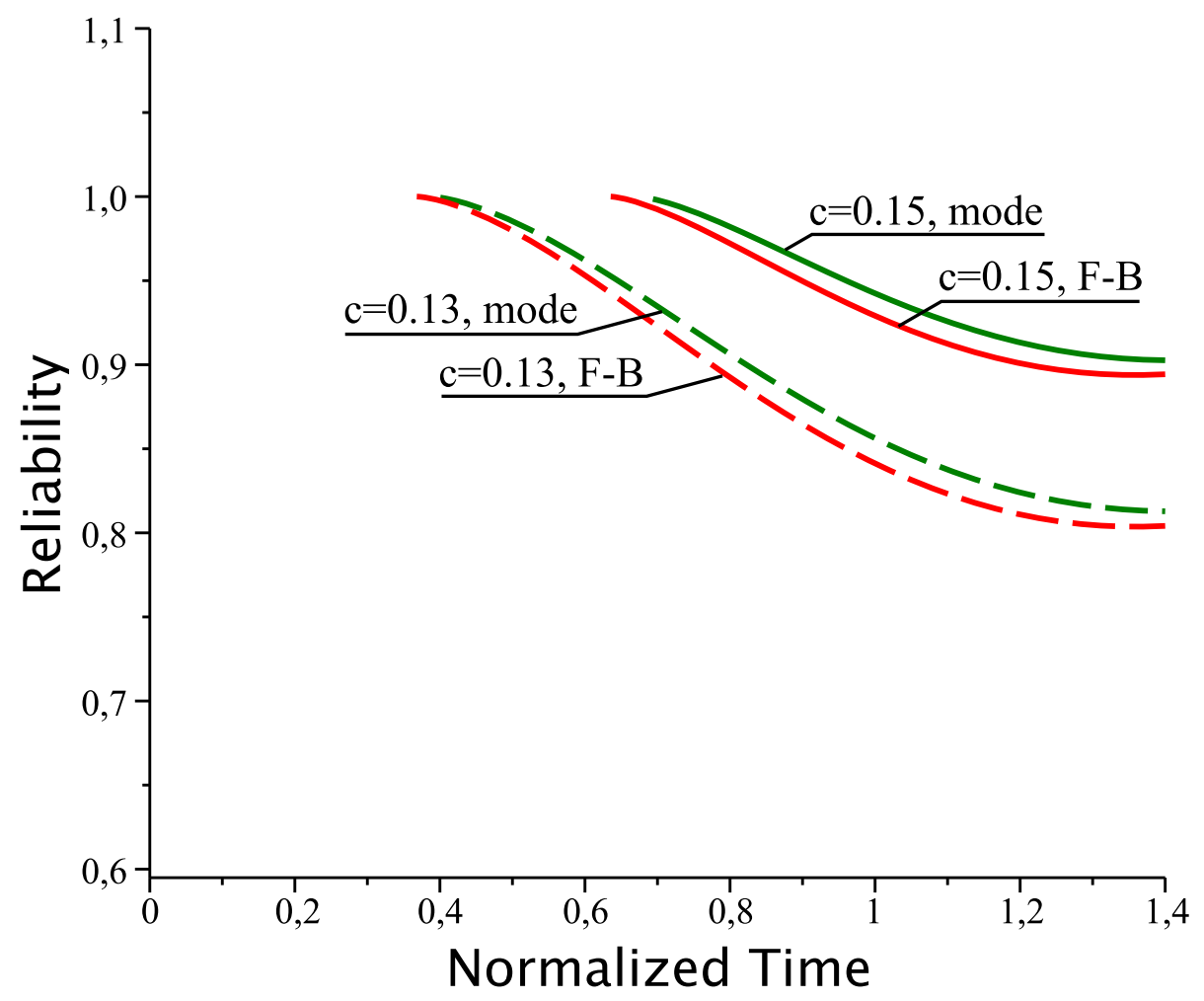

Figure 6: The reliability as a function of time $(\alpha=0.5)$. The green lines correspond to the solution with the normal modes; the red lines correspond to the solution with two term Filonenko-Borodich (F-B) approximation. 


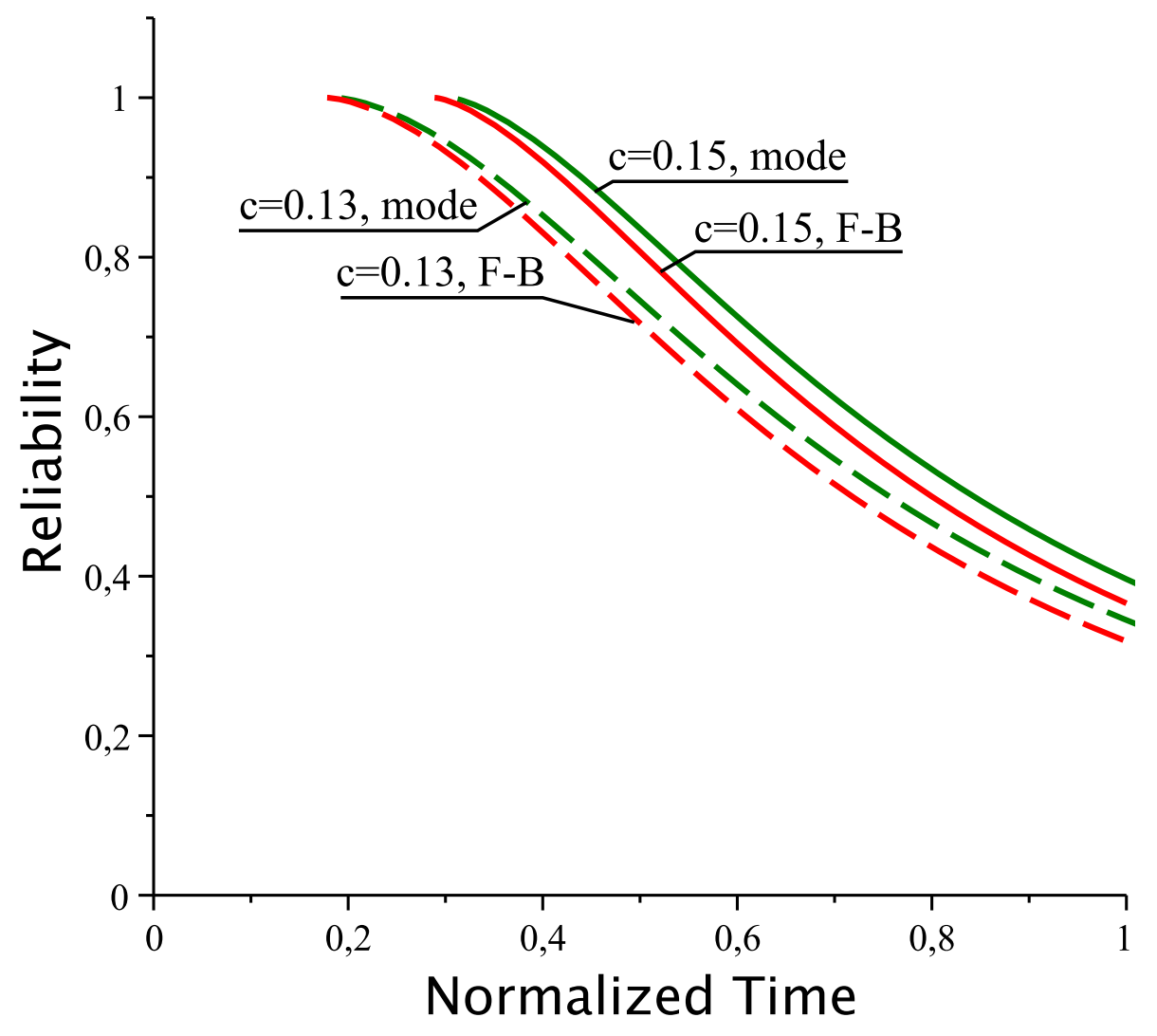

Figure 7: The reliability as a function of time $(\alpha=2)$. The green lines correspond to the solution with the normal modes; the red lines correspond to the solution with two term Filonenko-Borodich (F-B) approximation. 


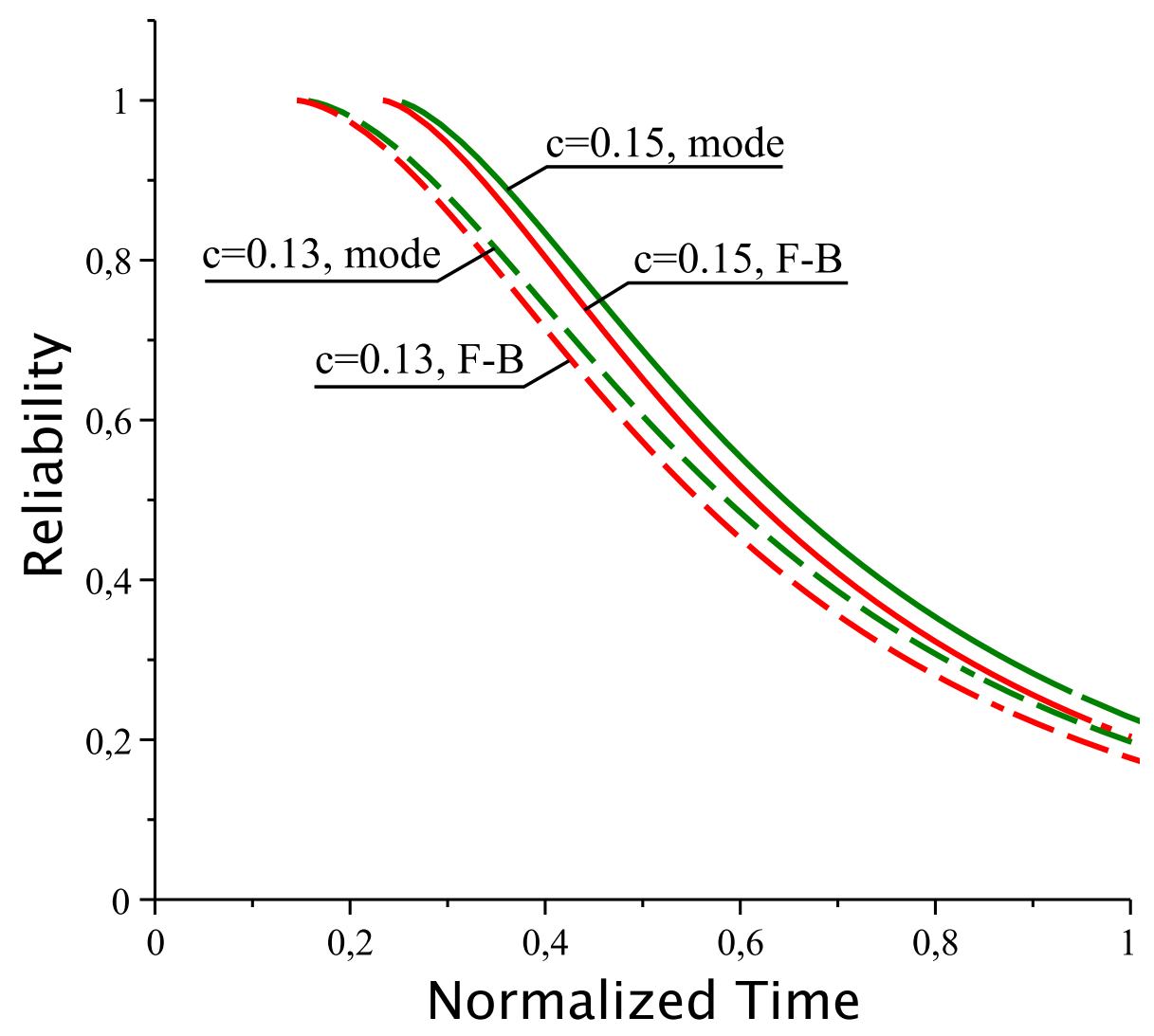

Figure 8: The reliability as a function of time $(\alpha=3)$. The green lines correspond to the solution with the normal modes; the red lines correspond to the solution with two term Filonenko-Borodich (F-B) approximation. 


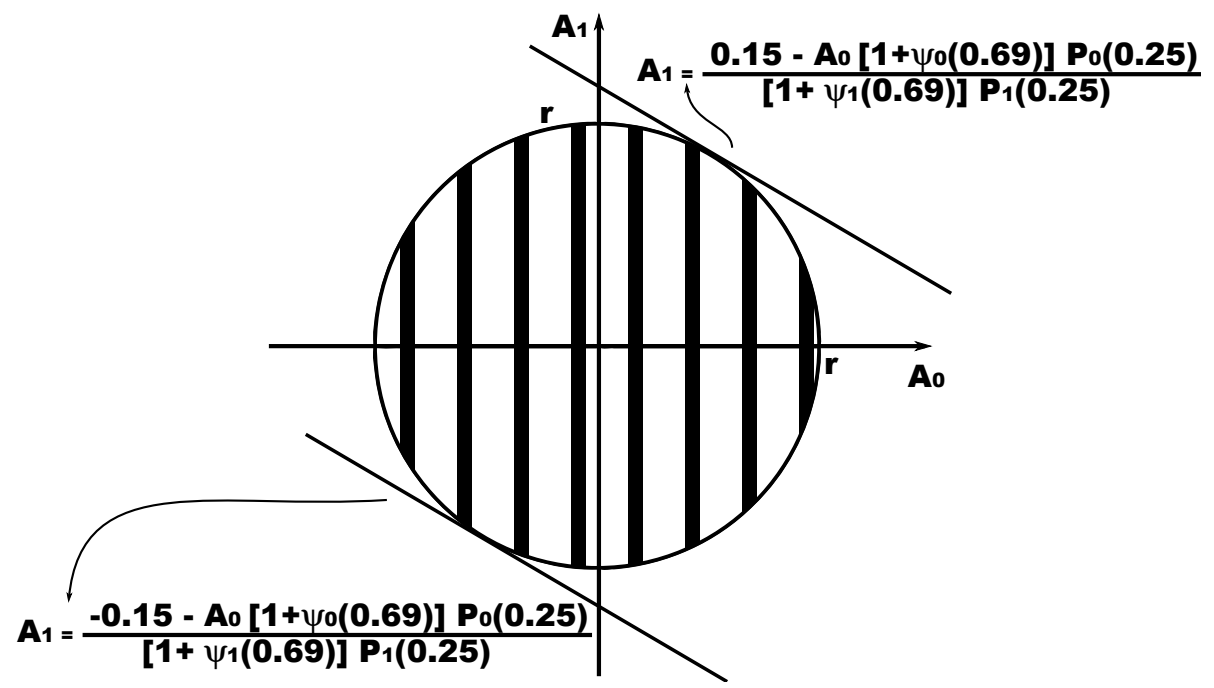

Figure 9: The area of integration of the probability density function for the reliability tending to one from below.

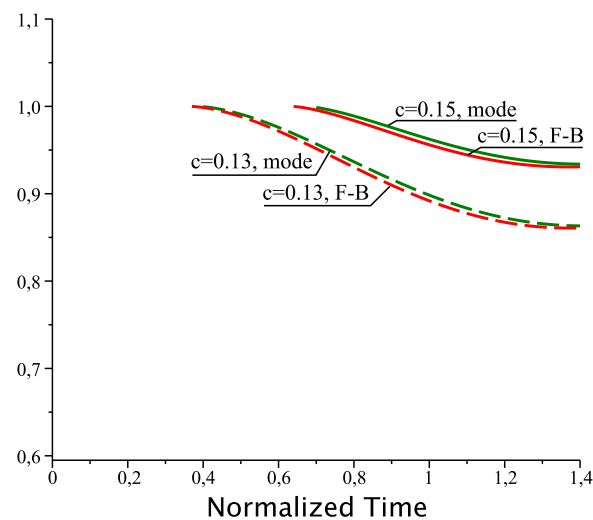

Figure 10: The reliability as a function of time $(\alpha=0.5)$. The green lines correspond to the solution with the normal modes; the red lines correspond to the solution with two term Filonenko-Borodich (F-B) approximation. 


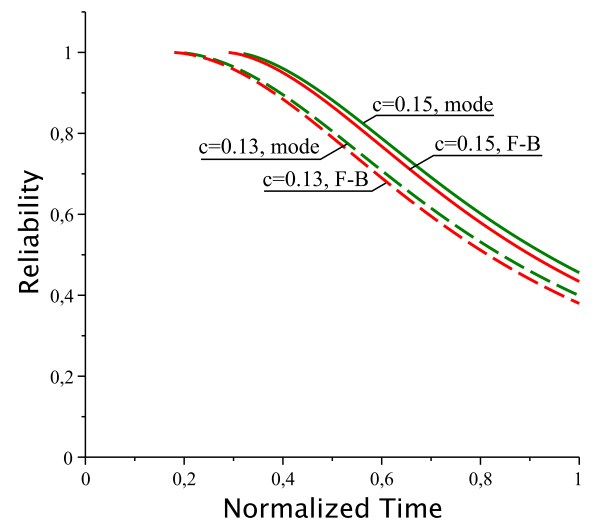

Figure 11: The reliability as a function of time $(\alpha=2)$. The green lines correspond to the solution with the normal modes; the red lines correspond to the solution with two term Filonenko-Borodich (F-B) approximation.

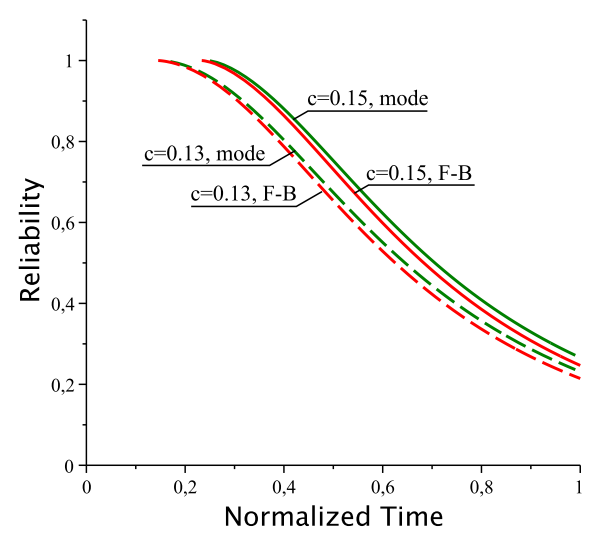

Figure 12: The reliability as a function of time $(\alpha=3)$. The green lines correspond to the solution with the normal modes; the red lines correspond to the solution with two term Filonenko-Borodich (F-B) approximation. 